\title{
Novel Translational Research Concepts and Strategies in Radiation Oncology
}

\author{
Seema Gupta ${ }^{1}$, P. K. Goyal ${ }^{2}$ and Mansoor M. Ahmed ${ }^{1}$ \\ ${ }^{1}$ Department of Radiation Oncology, University of Miami, Miami, Florida; ${ }^{2}$ Radiation \& Cancer \\ Biology Laboratory, Department of Zoology, University of Rajasthan, Jaipur, India
}

Meeting Report on International Conference on Radiation Biology and Translational Research in Radiation Oncology (ICRB-TRRO) held November 10-12, 2008 in Jaipur, India.

\begin{abstract}
The discovery of X-rays by Roentgen in 1895 and the discovery of radiations emitted from Uranium compounds by Becquerel led to the establishment of the field of Radiation Sciences. When Radiation Sciences utilized the knowledge in the field of Physics, Chemistry, Biology and Medicine, newer fields were established such as Radiation Oncology, -Chemistry, -Biology, -Protection, Physics, Radiology and Nuclear Medicine. Fields of Radiation Oncology and Radiation Biology have gained significant strides by embracing the modern concepts in molecular biology and physics of imaging. To discuss the recent trends and advances in these fields, Indian Society for Radiation Biology organized an "International Conference on Radiation Biology and Translational Research in Radiation Oncology (ICRB-TRRO)" in Jaipur, India during November 10-12, 2008. The primary theme of this international meeting was to focus on the translational research perspectives in radiation oncology using radiation biology findings as a reservoir of ideas. The second major theme was to focus on research initiatives and recent perspectives in radiation protection. A common dynamic theme constantly interacted with the primary and secondary themes was an influx of extensive discussion on the scientific utility and validity of novel agents obtained from different traditional systems of Indian medicine and herbal plant extracts that can be potent radio-sensitizers as well as potent radio-protectors (as countermeasure agents). Overall, four major thrust areas of the conference such as Radiation-induced signaling networks, novel therapeutics for cancer,
\end{abstract}

Received 05/10/09; accepted 05/18/09

Correspondence: Mansoor M. Ahmed, Ph.D. Department of Radiation Oncology, 1475 NW $12^{\text {th }}$ Ave (D-31), Miami, FL 33136, USA. Tel. 305-243-5454, Fax. 305-243-1854 e-mail: amansoor@med.miami.edu radiation-induced DNA damage-repair and novel imaging methods provided new insights and directions that had set roads to advancements in radiation biology.

Keywords: Apoptosis; Bystander effects; DNA repair; Energy balance; Imaging; Low dose fractionated radiation therapy

\section{Translational Research in Radiation Oncology}

The continual increase in the advancement of molecular biology coupled with the development of highly sensitive molecular techniques facilitate mushrooming of new focus areas of research in radiation biology, particularly, aimed at understanding the mechanistic processes that regulate radiation damage and response in both normal and tumor tissues. The fields of stem cell biology, nanotechnology and precision bio-functional imaging is rapidly transforming the translational research approaches to the field of radiation oncology. The challenge to radiation biologists and radiation oncologists is to use these cutting edge technologies to significantly improve the curative index in the management of human solid tumors by means of translating these new findings in the clinic. The International Conference on Translational Research in Radiation Oncology focused on these pertinent topics, to provide latest research information on the various novel strategies with the goal of increasing the therapeutic ratio through two broad areas: (1) the manipulation of tumor control by modulating the processes that control cell cycle regulation and apoptosis; and (2) the reduction of normal tissue morbidity by applying the emerging information on the molecular mechanistic basis of radiation sensitivity. It is evident from the literature that apoptosis is a minor component of cell killing in 
response to the clinical effects of radiation. However, if apoptosis is altered by inhibition or complete abrogation of anti-apoptotic factors that are often induced by clinical doses of radiation itself, then it may be possible to produce a conditional proapoptotic phenotype shift within the tumor cell population via altering the balance between pro- and anti-apoptotic events. Hence, molecular signalingbased apoptosis induction therapy will abrogate the relative resistance of tumor cells to radiationinduced apoptosis, and further enhance the therapeutic index in the management of human solid tumors. The current in-depth understanding of the cell cycle regulation in both normal and tumor cells paved insights into the mechanism of action of chemotherapeutic or biologic modifying agents that can act as radio-sensitizers. Further, the presence of aberrant cell cycle checkpoints in some cancers can be exploited to enhance the therapeutic index, specifically by the use of low-doses of radiation as potentiator of chemotherapy or biological modifiers. Lastly, increase in effective tumor control will be translated into clinics by the use of such treatments that do not cause deleterious effects in the critical normal tissue regions. Thus, the International Conference on Radiation Biology and Translational Research in Radiation Oncology provided a broad platform of opportunities for radiation oncologists, radiation biologists and medical physicists to discuss, interact and establish novel hypotheses, further test these hypotheses in laboratory settings in order to implement newer treatment strategies in the clinic.

\section{Radiation-induced signaling networks}

Nuclear and radiation issues are now front and center in public eye ranging from nuclear power to nuclear weapons \& nuclear/radiological terrorism. Radiation therapy and its benefits for patients with cancer have raised the public enthusiasm for the new technologies on the one hand and examination of their cost on the other. The advances in the basic radiation sciences are stunting. The elucidation of the molecular pathways of radiation effects may lead to novel biomarkers for radiation sensitivity, new tools for biodosimetry, ideal radioprotectors and sensitizers and improved multi-modality cancer therapy.

A typical tumor has compartments of normal tissue margins, tumor epithelium and neo-vascular endothelium. For gaining optimal effects of radiotherapy, it is highly important to understand the areas pertaining to the biology of tumor epithelium with respect to gene signaling depended growth, biology of tumor neo-vascularization under the regulation of gene expression and the biology of the bystander normal tissue around the tumor. These understandings will not only help to achieve enhanced tumor response but will significantly reduce normal tissue injury. This international conference provided a platform for such interactive topics where many unanswered questions were explored. Two symposia were organized to elaborate on the various signaling mechanisms induced by radiation leading either to apoptosis or therapy resistance.

In the symposium on Experimental Therapeutics: Apoptosis, Genotoxicity \& Cytotoxicity, Dr. R. Muschel, Oxford University (UK) proposed new models for metastasis in which different organs lead to vascular supply in different ways. These models could allow the development of therapeutic targets against metastasis. Dr. M. Saeed Sheikh, Upstate Medical University, Syracuse (USA) proposed that p53 and DNA damage regulated gene (PDRG) is an important mediator of genotoxic stress response and alterations in PDRG expression and function could be critically linked to pathobiology of human malignancies. Dr. Ying Huang, Upstate Medical University, Syracuse (USA) highlighted the importance of DOC45, a novel molecule that is linked to cellular stress response and growth regulation and also act as a valuable tumor marker. Dr. Gayathri Devi, Duke University, Syracuse Medical Center, Durham (USA) proposed a high throughput assay to analyze the effects of therapeutic agents in cells overexpressing specific inhibitor of apoptosis proteins (IAPs) and further develop an apoptotic signature of IAPs in normal vs. cancer cells. These cellular models will allow the initiation of a critical comparison of various IAP inhibitors towards the development of an optimal therapeutic agent for clinical trials against prostate, ovarian, liver and breast cancer.

In the other symposium on signaling networks and therapy resistance, Dr. E. Bernhard, Oxford University, Oxford (UK) talked on inhibition of oncogenic signaling, radiation sensitization and vascular normalization. He pointed out that the inhibition of tumor cell signaling at multiple points in the EGFR to AKT pathway results in prolonged improvement in tumor vasculature perfusion and decreased hypoxia. According to him, targeting this signaling pathway concurrent to treatment may benefit radiotherapy by enhancing tumor oxygenation. Dr. Mansoor M. Ahmed, University of Miami (USA) delivered talk on regulation of 
radiation -induced signal transduction by EGR-1 in prostate cancer. He mentioned that EGR-1 interacts with WW-domain containing protein YAP1 in response to radiation and EGR-1 function is a critical determinant of radiation response in prostate tumors. Dr. S. K. Apte, BARC, Mumbai (India), suggested an association between dessication and ionization radiation and offered an attractive possibility of use of engineered photoautotrophic Anabaena for biotechnological application in stressful environments. In another interesting talk by Dr. E. Sage, Orsay (France), it was observed that the inhibition of repair occurring at multiply damaged sites (MDS) in DNA is an important mechanism that prevents or limits the formation of deleterious repair intermediates like double strand breaks (DSB). On the other hand, it dramatically enhances the probability to get point mutation, instead, a price to be paid for survival.

\section{Novel therapeutics for cancer}

Radiation therapy has long been an integral part for the treatment and management of cancer. This session focused on the novel methods of cancer therapy that utilizes radiation to enhance its killing effects. Novel strategies like low dose fractionated radiation therapy (LDFRT), low and high dose radiation-induced bystander effects, glycolytic inhibitors, natural products as radiosensitizers were discussed to improve cancer therapy.

\section{Low dose radiation therapy}

Although, we have an understanding of the mechanism of cell death by radiation at conventional doses (1.8-2 Gy per fraction), our understanding of radiation effects at lower doses $(<1 \mathrm{~Gy})$ is still limited. The initial slope of the radiation cellsurvival curve (doses of 0-100 cGy) was presumed to be an ineffective dose range for human tumor therapy. However, as techniques to adequately study low dose radiation have improved, quite the opposite effect has been described. Joiner and colleagues revolutionized thinking about low doses of radiation ( $<100 \mathrm{cGy})$ by demonstrating an initial phase of hyper radiation sensitivity (HRS) to radiation using doses from 0 to 50 cGy $(1,2)$. To exploit the enhanced cell killing of low dose radiation without allowing proliferation would be to combine it with chemotherapeutic or biological modifier drugs. Thus, the role of LDFRT, as a chemotherapy potentiator in overcoming intrinsic chemotherapy resistance is a novel concept. Dr. Seema Gupta, University of Miami, Florida (USA) discussed this novel strategy of chemopotentiation of the effects of cisplatin by LDFRT in lung cancer cells in a symposium on low dose radiation effects: currents trends \& translational perspectives. Findings from her work indicated that the chemopotentiation by LDFRT is correlated more with the intrinsic radiation sensitivity of the cells rather than hyper-radiation sensitivity phenomenon while the mode of cell killing is either through apoptosis or clonogenic inhibition or both in lung cancer cells. Dr. Eduoard Azzam, University of Medicine and Dentistry, Newark (USA) discussed about low-dose ionizing radiation-induced bystander effects mediated mechanisms and impact on health risks. Dr. Carmell Mothersill, Mc Master University, Ontario (Canada) discussed radiation-induced stress effects following low dose exposure. Her presentation added the knowledge about low dose radiobiological effects in both human and nonhuman biota. Dr. Marc Mendonca, Indiana University, Indianapolis (USA) delivered the talk on tumor suppressor gene (FRA-1) in radiation-induced carcinogenesis. Dr. Amy Kronenberg, Lawrence Berkeley National Laboratory, Berkeley (USA) discussed about the current understanding of the role of DNA double strand breaks in radiation mutagenesis and in the prevention of autosomal mutagenesis. She also highlighted the role of antiapoptotic members of the BCL-2 family in modulating DNA double strand break repair.

\section{Bystander effects in radiotherapy}

Bystander effects are defined as effects occurring in tissues not themselves irradiated directly. Following high dose therapy of tumors such effects include collateral damage to normal tissue surrounding the tumor or tissue receiving signals carried by blood or resulting from scatter. The issues are whether such signals are protective, resulting in the induction of adaptive or restorative processes, or destructive due to increasing the normal tissue target volume. The issue of therapeutic ratio is important here too in the light of reports that tumors may not produce toxic or death-inducing bystander signals while normal tissues certainly can. In a symposium on Translational research in Radiation Oncology: Development of novel radiation therapeutic strategies, Dr. Mohammed Mohiuddin from Geisinger- Fox Chase Cancer Center, WilkesBarre, PA (USA) delivered a talk on spatially fractionated grid radiation (SFGRT) in the management of advanced cancers. He confirmed the efficacy and safety of using SFGRT radiation in 
patients with bulky tumors as this can improve local control of disease and achieve long term survival in patients where conventional treatment alone has a limited chance of success. These responses may be mediated by bystander and abscopal effects of high dose radiation.

Following low-dose radiation imaging-type exposures, the issues are whether for example doses associated with CAT scans or x-rays can be harmful by triggering bystander effects leading to genomic instability or whether they are actually beneficial, leading to adaptive and restorative responses.

Dr Gillis McKenna, Oxford University (UK) presented a talk on searching for a consensus target for radiosensitivity which was focused on the alterations in expression or activation of signal transduction pathways as hallmarks of cancer. Stephan Hahn, University of Pennsylvania, PA (USA), also discussed about the relationship between the tumor microenvironment/hypoxia and activation of different signaling pathways. Dr. Georg Bauer, Universitatsklinikum, Friedburg (Germany) specified the nature of low dose radiation induced bystander effects on specific signaling components of intercellular induction of apoptosis at defined stages of multistep carcinogenesis. Whereas low dose radiation has a strong direct impact on transformed cells (early stages of multistage carcinogenesis), its impact on late stage tumor cells depends on the presence of secondary plant compounds. These findings may have implications for the understanding of the control of carcinogenesis by low dose radiation as well as for the interaction of food-derived plant products and low dose radiation. $\mathrm{He}$ also suggested to utilize the underlying reactive oxygen species signaling chemistry for novel therapeutic approaches based on low dose radiation.

\section{Targeting of glucose metabolism}

Development of an approach based on the inhibition/modulation of glycolysis linked cell signaling using 2-deoxy-D-glucose (2-DG), a glycolytic inhibitor that exploits differences in the status of glucose dependent energy metabolism between tumor and normal tissues has shown promising results. 2-DG enhances the sensitivity of tumor cells to radiation and chemotherapeutic drugs in vitro and in vivo and simultaneously reduce the radiation-induced damage in normal cells and is currently in Phase III clinical trials for glioma patients $(3,4)$. A symposium was organized that focused on targeting glucose metabolism for improving radiotherapy \& chemotherapy of cancer.
It was preluded by Dr. Viney Jain, Delhi (India) who reviewed the importance of energy metabolism and its exploitation for cancer therapy. Studies from Dr. B.S. Dwarakanath, INMAS, Delhi (India) group concluded that alterations in cell signaling linked to tumor physiology and host tumor interactions contribute significantly to the radio- and chemosensitization of tumors by 2-DG. Further, he suggested that in vitro multicellular tumor spheroids model could be very useful in predicting responses of in vivo tumors to bioenergetics based therapeutic modalities besides providing insight into the possible mechanisms involved. Dr. D. Spitz, University of Iowa, Iowa (USA) described the applications of findings from his group that cancer cells may have a defect in mitochondrial respiration leading to increased levels of reactive oxygen species and glucose metabolism may be increased to provide reducing equivalents to compensate for this defect to develop new combined modalities for cancer therapy. $\mathrm{He}$ also elaborated on the clinical implications for FDG-PET imaging to predict tumor responses to therapy. Dr. Sarin, Tata Memorial Hospital, Mumbai (India) described the clinical utilization of 2-DG with hypofractionated radiation therapy for Glioblastoma Multiforme (GBM) patients. He concluded that this novel therapeutic approach has the potential to improve the survival and quality of life in GBM and merits further clinical evaluation in other human cancers.

\section{Natural agents as radiation sensitizers}

In recent times, extensive search for complimentary alternative medicine (CAM) products in treatment of various non-curable diseases is underway. Plant based products such as Taxol made a remarkable impact in the management of several solid tumors. Thus, identification of newer herbal agents as radio-sensitizers and radio-protectors is warranted. This international conference provided unique opportunity to focus on several indigenous medicine systems that originally mushroomed in Indian subcontinent and discuss the potential of several herbal agents as radiation sensitizers and radiation protectors.

A number of traditional systems of medicine exist in India for more than 3000 years that include Siddha, Ayurveda and Unani medicine. Two herbal agents Curcumin and Withaferin $A$ that is abundantly used in the Indian systems of medicine have been demonstrated to be potent radiosensitizers. Several other herbal agents have reported to have potent anti-cancer activity (5-19). 
Many laboratories and biotech companies are working in the development of new herbal agents as anti-cancer drugs.

Two symposia were targeted on Radiation response modulation by natural or/ chemical compounds, plant products \& antioxidants. Dr. Nagraj Huilgol from Dr. Balabhai Nanavati Hospital, Mumbai (India) delivered a talk on issues in clinical radiation protector - A fresh look at old molecules for an old problem in which he urged upon a fresh look at the earlier molecules and need for exploring novel drug delivery methods so that toxicities associated with chemo radiotherapy can be reduced. Dr. S.S. Lahiri, INMAS, Delhi (India), Dr. Madhubala, INMAS, Delhi (India), Dr. Manju Lata Gupta, INMAS, Delhi (India) and Dr. F. Domann, University of Iowa (USA) discussed the promising role of neutraceuticals and various botanicals for protection against radiation and oxidative stress. Dr. B. N. Pandey, BARC, Mumbai (India) talked about role of cellular oxidative stress and apoptotic death in enhancement of tumor radio-sensitivity by natural plant products.

\section{New radiotherapy approaches}

A symposium was arranged on new radiotherapy approaches and treatment modalities. Dr. Harold M. Swartz, Dartmouth Medical School, Hanover (USA) delivered a lecture on optimizing radiation therapy by directed repeated measurements of oxygen in tumors in patients. He discussed about quantitative measurements of pO2 during radio- and chemotherapy which helps the physicians in the characterization of status of disease and the effects of therapeutic measures during cancer treatment. Dr. A. Chougule, SMS, Jaipur, (India) and Dr. N. Patni, BMCHRI, Jaipur (India) discussed about developments in India and impact of concurrent chemo-radiation in locally advanced uterine-cervix carcinoma. Dr. Shruti Jolly, University of Michigan (USA) presented a talk on the evolving role of targeted therapy in the treatment of head and neck cancer. Dr. Oliver Guipaud, Institute for Radioprotection \& Nuclear Safety (France), focused on investigation of the serum protease to look for diagnostic and prognostic biomarkers of ionizing radiation.

Drugs that interfere with the process of angiogenesis in tumors are of increasing interest in combination treatments but because of the potential for inducing hypoxia there is concern that their use with radiation will require careful timing. Further, recent studies have suggested that radiation damage to endothelial cells can cause their apoptosis and play an important role in response of tumors and tissues to radiation treatment (20). Of particular interest, this effect on endothelial cells seems to be dependent on the size of the radiation dose with effective doses being larger than conventional-sized fractions but rather in the range of doses used in stereotactic radiation treatments of brain or, increasingly, other sites in the body such as lung. There is a possibility that hypoxia caused by vascular damage could play an important role in tumor radiation response using such larger doses. The deliberations by Indian and foreign scientists addressed the current state of knowledge in these related areas in North America and India and discussed both the underlying mechanisms of these effects and how they may be optimally exploited for improved therapeutic outcome. Dr. Brown, Stanford University School of Medicine, Stanford, CA (USA) suggested that although the sensitivity of the tumor vasculature affects the growth delay of irradiated tumors it does not affect the dose to cure $50 \%$ of tumors (TCD50) because bone marrow derived cells can "rescue" the tumor vasculature. However, if the bone marrow contribution is inhibited, tumors can be permanently controlled by considerably lower radiation doses than is usually the case.

\section{Radiation-induced DNA damage and repair}

The most important and obvious phenomena induced by radiation is DNA damage. It is highly important to understand how cellular repair to the radiation-induced damage influence the response and what are the mechanisms involved so that these pathways can be targeted.

In the symposium, DNA Repair in the clinic, Dr. Simon Powell, Memorial Sloan-Kettering Cancer Center, New York (USA), delivered a talk on "Defining a BRACA1/BRACA2 dependent pathways of DNA repair in human breast cancer". In his talk, he emphasized that functional assays of BRACA 1 and 2 could become a useful technique to determine phenotype of human breast cancer, which influences the choice of therapy. Dr. Tej K. Pandita, Washington University, St. Louis (USA) discussed about histone code and DNA damage response while Dr. Kumkum Khanna from Queensland Institute of Medical Research, Herston (Australia) presented her lecture on involvement of novel single-stranded DNA binding protein in DNA damage response and genomic stability. She has functionally identified and characterized two novel single stranded DNA binding proteins, hSSB1, and hSSB2, which are over 
expressed in many forms of cancer and might turn out to be ideal targets for treatment of cancer. Dr. R. C. Chaubey, BARC, Mumbai (India) described the comet assay as a sensitive assay to quantify DNA damage and suggested that it may be useful to predict the cellular radiosensitivity of tumor cells.

\section{Novel imaging techniques}

Novel imaging techniques are being developed not only for diagnosis but also for therapeutic purposes. Nanotechnology is the most exciting new field that is being increasingly utilized for imaging using fluorescent compounds tagged to the nanoparticles. In vivo molecular imaging continues to grow as a significant new technology that provides unmatched real time and longitudinal research capabilities in small animals. Continued improvements in signal localization, quantitation, throughput, repeatability, sensitivity and ease of use are required in molecular imaging systems and imaging agents to allow researchers to maximize the utility of this exciting technology.

One symposium was targeted on Molecular imaging and nanotherapeutics in radiation therapy. Dr. Rao Papineni and Dr. William E. McLaughin, Carestream Health Inc. (USA) reviewed the state of the art Kodak Multispectral FX imaging system and Kodak X-sight Imaging Agents with an emphasis on the use of multispectral and multimodal -Optical, Xray and Radioisotopic- imaging to provide an improved understanding of the localization and quantitation of both anatomical and molecular imaging signals in small animals. Dr. Mark Kester, Pennsylvania State University (USA) described the promise of bioresorbable, indocyanine green-doped, calcium phosphate nanoparticles for near-infrared in vivo diagnostic imaging of human breast cancer that may help in the early detection of solid tumors. Dr. A. K. Mishra, INMAS, Delhi (India) discussed about design and synthesis of methionine conjugated novel spect-pharmaceutical for targeted imaging and therapy of methionine dependent human tumors. These presentations were mainly focused to introduce radiation oncology clinicians and researchers to the potential application of recent advances in nanotechnology to the realm of radiation oncology.

\section{Radiation-induced genomic instability}

In the symposium on Radiation induced genomic instability, Dr. G. Iliakis from University of Duisburg, Essen Medical School (Germany) delivered a talk on backup pathway of DNA double strand break repair in cells of higher eukaryotes. Dr. Dudley Goodhead, MRC (UK) presented his talk on consideration of radiation quality, dose and targets for induced genomic instability. He mentioned that it is an important area of study, with potential implications, not only for cancer risks, but also for other health effects. Dr. Munira Kadhim from Oxford Brookes University, Oxford (UK), delivered her talk on genetic and dose effect on radiationinduced genomic instability. She concluded that her results may have implications for human health and radiation protection standards in particular at environmentally relevant exposures.

In addition to these focus areas, the conference also provided an opportunity to understand the protective effects of radiation. The risk of military, civilian, and emergency personnel being exposed to lethal doses of ionizing radiation is increasing. Nuclear proliferation, terrorist activity, and the distribution of nuclear and radioactive materials through underground networks make incidents involving radiation injuries increasingly likely. Scenarios involving radiological hazards include nuclear detonations, covert placement of radioactive substances, and dirty bombs. The other risk from radiation exposure can be from accidental exposures during radiation therapy or use of radioisotopes for diagnosis purpose. Thus, based on the exposure, radiation hazards can vary from late life pathologies to acute mortality. The severity of radiation-induced complications is directly proportional to the dose of radiation. Disease and mortality in the treatable range are largely due to the hematopoietic syndrome, and, at higher doses, the GI syndrome. Thus, as is recognized by both civilian and military agencies, the need for new countermeasures is urgent in the management of such radiation-induced acute severe symptoms and as well as for late effects. The International Conference focused on pertinent topics, to provide latest research information on the various novel strategies and mechanisms with the goal of providing novel ideas through two broad areas: (1) the manipulation of signaling pathways to develop novel therapeutic countermeasure for acute radiation injury; and (2) underlying molecular mechanisms of adaptive response for chronic low-dose exposures. Thus, this international conference provided a platform for both experts and novice in the field to interact and develop collaborative research approaches in discovering and testing newer countermeasures of radiation injury. 


\section{Radiation Countermeasures}

The development of safer and more effective radiation countermeasures is critical for protecting civilians and military personnel from unintended radiation exposure in this currently heightened nuclear threat environment. Radiation countermeasures are needed to protect personnel not only from early arising (acute radiation syndrome) effects, but from late arising pathologies (e.g., lung fibrosis, cancer) as well. The U.S. Food and Drug Administration (FDA) have approved no safe and effective radiation countermeasures for human use in a non-clinical setting. Several compounds are in different stages of evaluation, but so far none possesses all the requisite qualities to be an optimal radiation countermeasure. Drugs are needed that can be given before irradiation (radioprotectants) and after exposure (mitigators).

To be a useful countermeasure against radiation, an agent must fulfill the following criteria: it should be effective, safe (free of side effects, drug interactions), stable and easy to stockpile, have no abuse potential, be useful in different operational environments (military and civilian), be stable in the field, be useful in combined injury, and have a suitable timing of administration. Realization of these facts has caused a shift in emphasis away from agents that interfere directly with the chemical effects of radiation, such as free radical scavengers, to compounds that target the pathophysiological manifestations of radiation injury or boost the immune response (immunomodulators). Currently, several agents with immunomodulatory potential, cytokines, growth factors, and statins, are under investigation.

A special session was dedicated to discuss on nuclear disaster management \& countermeasures initiatives. Dr. C. Norman Coleman, Department of Health \& Human Services (USA) delivered a talk on radiological and nuclear terrorism in which he presented an overview of integrating radiation biology, physics and medical countermeasures into national and International response planning. Dr. Vadim Krivokrysenko, Cleveland Biolabs, Buffalo (USA) discussed that CBLB502 is a novel non-toxic radiation countermeasure that reduces radiation damage when administered in prophylactic and mitigating regimens to the hematopoietic and G.I. tract, and therefore has a great potential as a life saving treatment. Several other speakers discussed various methods and strategies for medical countermeasures for nuclear disaster management. The need was assessed to ensure implementation of
National Disaster Management Authority plans/guidelines and coordination between various services providers to manage radiological and nuclear emergencies in an effective manner.

In addition, one symposium on radiation medical countermeasures and emergency preparedness was sponsored by National Institute of Allergy \& Infectious Diseases (NIAID) (USA). It included the speakers Dr. Narayani Ramakrishnan, NIH (USA), Dr. William Mc. Bride, University of California, LA (USA), Dr. Thomas Mac Vittie, University of Maryland, Baltimore (USA) and Dr. Solomen Paul, SRM Universitry, Chennai (India). Dr. N. Ramakrishnan delivered the talk on Medical countermeasures of radiation injury in which she described the programs sponsored by NIAID for the product development as medical countermeasure against intentional and/or accidental radiation exposure. Dr. McBride delivered a talk on "Can radiation response modifiers be profiled by high throughput screening of small molecules libraries". He explained the mechanism through which cellular response to irradiation can be modified in vitro and in vivo by application of numerous agents. Dr. Thomas Mac Vittie described the medical countermeasures against the acute hematopoietic and gastro-intestinal syndromes: best available today. He suggested TBI/BMS models for effective medical management for lethally irradiated personal in the aftermath of a nuclear terrorist event. Dr. M.H. Jenson (USA) made his presentation on Gastro-intestinal radiation injury: significance, mechanisms and countermeasures where he dealt with the clinical significance and pathogenesis of intestinal radiation toxicity and post-radiation medical countermeasures which are currently under development to mitigate such radio-lesions. In addition, results from Dr. P. K. Goyal, Rajasthan University, Jaipur (India)'s group demonstrated that extracts from several medicinal plants and phytochemicals can serve as novel radioprotectors for medical countermeasures against radiation injury.

\section{Molecular Basis of Radioprotection}

At the organism level, the immediate effects of low- and moderate-intensity radiation are largely caused by cell death and bystander effects. Hematopoietic (HP) and gastrointestinal (GI) radiation syndromes are the cause of short-term radiation-induced lethality at higher doses. HP syndrome is caused by the loss of blood cells and their progenitors making it impossible to regenerate 
blood and the lymphoid system, leading to death from hemorrhage, anemia and infection (results from immunosuppression). GI syndrome is caused by massive cell death in the intestinal epithelium, predominantly in the small intestine, followed by disintegration of the intestinal wall and death from bacteriemia and sepsis.

During late $90 \mathrm{~s}$, several researchers found that molecular mechanisms of radiation-induced apoptosis in sensitive tissues, including HP, lymphoid tissues, hair follicles and epithelia of the gut, is mediated by activation of the tumor suppressor protein p53. Genetic suppression of p53 makes animals resistant to radiation-induced HP syndrome and alopecia. Several reports established inhibition of apoptosis as an important line of protection from radiation. Although suppression of p53 was shown effective in protection from HP syndrome, it appeared to have several important limitations (probable carcinogenic effects, epithelial cell growth arrest - mitotic catastrophe in GI). Activation of $\mathrm{NF}_{\kappa} \mathrm{B}$ pathway is considered as a novel strategy. The protective role of $\mathrm{NF} \kappa \mathrm{B}$ is at least threefold and is mediated via transcriptional activation of multiple genes coding for: a) antiapoptotic proteins that block both major apoptotic pathways, b) cytokines and growth factors that induce proliferation and survival of HP and other stem cells, and c) potent ROS-scavenging antioxidant proteins, such as MnSOD (SOD-2). In brief, current approaches target molecular signaling pathways involved in radiation injury and recovery.

Dr. Elizabeth Travis from MD Anderson Cancer Center, Houston (USA) presented her lecture on targeting radioprotector to the lung using genetic approaches where she mentioned about most recent data available on the genetics of radiation induced fibrosis and pneumonitis. Dr. Andrei Gudkov from Roswell Park Cancer Institute, Buffalo (USA) presented his talk on protecting stem cells from ionizing radiation by pharmacological modulation of major death/survival pathways. He emphasized on the pharmacological modulation of $\mathrm{p} 53$ and NF- $\mathrm{\kappa B}$ pathways mimicking the modes of their degeneration in tumor cells is a powerful approach to radioprotection. Dr. Mark Whittnall, US Armed Forces Radiobiology Research Institute (AFRRI), Bethesda (USA) discussed about radiation countermeasures research at the AFRRI. Dr. Vijay K. Singh from the same institute described cytokines as biomarkers for efficacy of radiation countermeasures. Dr. K. B. Sainis, BARC, Mumbai (India) focused his talk on differences in low dose radiation-induced immunomodulation and gene expression in two strains of bred mice. Dr. A. Wahab, University of Miami (USA) discussed in her talk about the use of antagonists of growth hormone releasing hormones for protection during whole-body radiation.

\section{Summary}

The International Conference on Radiation Biology \& Translational Research in Radiation Oncology provided an active and vibrant forum for sharing new results, ideas and a great deal of stimulating discussions among spectrum of researchers in radiation biology and radiation cancer therapy clinicians. It is expected that the deliberations and recommendations of conference would help in formulating newer concepts/strategies by basic and applied radiation scientists as well as cancer researchers and even for the policy makers. Overall, the scientific deliberations during the conference will certainly generate many collaborations between Indian and foreign faculty as well as young and senior scientists/clinicians. It is hoped that this forum can help the field of radiation research to garner the necessary resources for research, technological development, radiation protection and clinical care by linking science with societal needs in a manner understandable to both the founders and potential users. The complexity of the radiological sciences, technology and socioeconomic issues were best addressed through multidisciplinary collaborative approaches during this conference that will bring the scientists and clinicians together to address the challenges and opportunities in radiation and cancer research. In the forthcoming issues of Molecular and Cellular Pharmacology selected presentations from this international conference will be featured as fulllength articles.

\section{Acknowledgments}

This conference was supported by the Indian Government agencies (Indian Council for Medical Research, Department of Science and Technology, Medical Council of India, Department of Atomic Energy-BNRS, Indian National Science Academy, National Disaster Management Agency and Central Scientific and Industrial Research); US Federal agencies (NIH-NAID, Department of Energy); Societies (International Association of Radiation Research, Radiation Research Society, American Society for Therapeutic Radiation Oncology) and Companies (Varian, Kodak's Carestream Health, Seimens-India, Dr Reddy's Laboratory). 


\section{References}

1. Joiner MC. Induced radioresistance: an overview and historical perspective. Int J Radiat Biol 1994;65:79-84.

2. Marples B, Lambin P, Skov KA, Joiner MC. Low dose hyper-radiosensitivity and increased radioresistance in mammalian cells. Int J Radiat Biol 1997;71:721-35.

3. Dwarkanath BS, Jain VK. Energy linked modifications of the radiation response in a human cerebral glioma cell line. Int J Radiat Oncol Biol Phys 1989;17:1033-40.

4. Gupta S, Mathur R, Dwarakanath BS. The glycolytic inhibitor 2-deoxy-D-glucose enhances the efficacy of etoposide in ehrlich ascites tumor-bearing mice. Cancer Biol Ther 2005;4:87-94.

5. Arathi G, Sachdanandam P. Therapeutic effect of Semecarpus anacardium Linn. nut milk extract on carbohydrate metabolizing and mitochondrial TCA cycle and respiratory chain enzymes in mammary carcinoma rats. J Pharm Pharmacol 2003;55:1283-90.

6. Arora R, Gupta D, Chawla R, et al. Radioprotection by plant products: present status and future prospects. Phytother Res 2005;19:1-22.

7. Aslam M, Bano H, Vohora SB. Sartan (cancer) and its treatment in Unani Medicine. Am J Chin Med 1981;9:95107.

8. Go VL, Champaneria MC. The new world of medicine: prospecting for health. Nippon Naika Gakkai Zasshi 2002;91 Suppl:159-63.

9. Ichikawa H, Nakamura Y, Kashiwada Y, Aggarwal BB. Anticancer drugs designed by mother nature: ancient drugs but modern targets. Curr Pharm Des 2007;13:340016.

10. Naik GH, Priyadarsini KI, Satav JG, et al. Comparative antioxidant activity of individual herbal components used in Ayurvedic medicine. Phytochemistry 2003;63:97-104.

11. Nisa M, Akbar S, Tariq M, Hussain Z. Effect of Cuscuta chinensis water extract on 7,12dimethylbenz[a]anthracene-induced skin papillomas and carcinomas in mice. J Ethnopharmacol 1986;18:21-31.

12. Pal S, Chakraborti SK, Banerjee A, Mukerji B. Search for anti-cancer drugs from Indian medicinal plants (Ayurvedic, Unani, etc.). Indian J Med Res 1968;56:445-55. 13. Ranga RS, Girija R, Nur-e-Alam M, et al. Rasagenthi lehyam (RL) a novel complementary and alternative medicine for prostate cancer. Cancer Chemother Pharmacol 2004;54:7-15.

14. Shah ZA, Vohora SB. Antioxidant/restorative effects of calcined gold preparations used in Indian systems of medicine against global and focal models of ischaemia. Pharmacol Toxicol 2002;90:254-9.

15. Sowmyalakshmi S, Nur EAM, Akbarsha MA, Thirugnanam S, Rohr J, Chendil D. Investigation on Semecarpus Lehyam--a Siddha medicine for breast cancer. Planta 2005;220:910-8.

16. Sujatha V, Sachdanandam P. Recuperative effect of Semecarpus anacardium linn. nut milk extract on carbohydrate metabolizing enzymes in experimental mammary carcinoma-bearing rats. Phytother Res 2002;16 Suppl 1:S14-8.
17. Vayalil PK, Kuttan G, Kuttan R. Protective effects of Rasayanas on cyclophosphamide- and radiation-induced damage. J Altern Complement Med 2002;8:787-96.

18. Veena K, Shanthi P, Sachdanandam P. The biochemical alterations following administration of Kalpaamruthaa and Semecarpus anacardium in mammary carcinoma. Chem Biol Interact 2006;161:69-78. 19. Veena K, Shanthi P, Sachdanandam P. Therapeutic efficacy of Kalpaamruthaa on reactive oxygen/nitrogen species levels and antioxidative system in mammary carcinoma bearing rats. Mol Cell Biochem 2007;294:12735 .

20. Fuks Z, Kolesnick R. Engaging the vascular component of the tumor response. Cancer Cell 2005;8:8991. 